XXX.

Aus dem Institut für demonstrative Pathologie der Kgl. Universität Neapel (Director: P. F. Castellino).

\title{
Ueber die Wirkung der Herzmittel nach den neueren Lehren über die allgemeine Physiologie des Herzens.
} Klinische und experimentelle Untersuchungen.

Von

\author{
Prof. S. La Franea.
}

(Hierzu Tafel XY.)

Im Allgemeinen beruht die Behandlung der Herzkrankheiten, soweit sie die Herzfunction wieder herstellen will, auf den durch das Studium der Hämodynamik gelieferten Daten, welche die meisten Autoren theoretisch nicht nur durch die im Organismus entstandenen Verhältnisse, sondern vorzugsweise dureh die Natur der Affection oder den Herzfehler, der daran schuld ist, erklären. Daher die Eintheilung in systolische und diastolische, hyper- und hypotensive Mittel, Herıtonica und Diuretica (Séé ${ }^{1}$ ), deren Indication nicht durch den Zustand bedingt sein soll, der das Einschreiten verlangt, sondern durch den, der die Affection verursacht hat; so hält man auch ein Arzneimittel bei einer bestimmten Affection für indicirt oder contraindicirt, während doch in Folge der Affection und während ihres Verlaufes so viele Bedingungen eintreten und zusammen wirken können, dass diese Präventivbehandlung unnütz, ja schädlich wird. Bei der klinischen Behandlung sind derartige Ansichten nur selten maassgebend und Rum $\mathrm{mo}^{2}$ ), um keine anderen Beispiele anzuführen, hat vor einer Reihe von Jahren mit Recht die Arzneimittel klassificirt, je nachdem sie auf den Nerven- oder auf den Muskelapparat einwirken; ihre richtige Anwendung ist jedoch nur dann möglich, wenn man genau weiss und beobachtet, welchen Antheil jeder der beiden Apparate im einzelnen Falle an der Herzaffection nimmt, und dies lässt sich, ausser bei den sogenannten Herzneurosen, kaum feststellen.

Wenn wir von den Störungen absehen, die in Folge des Einflusses der extracardialen Nervenapparate eintreten, ohne dass die Lebensfähigkeit des Herzens darunter leidet - mögen nun die verschiedenen Bakterien oder Gifte mit den durch sie verursachten entzündlichen oder degenerativen Zuständen einwirken oder Kreislaufstörungen im Herzen selbst zu Stande kommen, wie bei Klappenfehlern oder Veränderung der Coronararterien, so können wir sagen, dass die Störungen auftreten, wenn die Lebens-

1) Séć, La thérapeutique physiologique du coeur, Paris 1893.

2) Rummo, Riforma medica seit 1888 . 
fähigkeit der Herzmuskelfaser und ihrer Nervenapparate direct in Mitleidenschaft gezogen wird.

Von den "dyssistolischen" Erscheinungen (Leclereq ${ }^{1}$ ) an bis zu den unter dem Namen Hypo-, Hyper-, Asystolie zusammengefassten handelt es sich nur um Grade der darauf folgenden Aenderung ihrer Eigenschaften, durch welche die Mechanik des Herzens bedingt ist; deshalb muss man beim Studium, namentlich aber bei der Therapie der Herzaffectionen, nicht nur die Störungen der Hämodynamik und die daraus resultirenden Umstände, sondern vor allem den Zustand des Organs berücksichtigen. Denn welche Bedeutung kann man z. B. vom therapeutischen Gesichtspunkt aus der Natur (nicht dem Grad) eines Herzlehlers beimessen, wenn man die mangehafte Compensation bekämpfen muss? Letztere tritt nicht ein, weil der Febler besteht, von dessen Vorhandensoin vielleicht lange Zeit hindurch keine Symptome zu erkennen sind, sondern weil in Folge der veränderten Ernührung des Herzens (Abriahme des ErnährungsCoefficienten) oder in Folge der regressiven Veränderungen der Ganglienzellen des Herzens $(\mathrm{Ott})$ die Functionen der Herzfaserzellen oder des intracardialen Nervenapparates, oder auch beides gleichzeitig, nachlassen und das Herz nicht mehr im Stande, ist die in der Hydraulik des Kreislaufes eingetretenen abnormon Bedingungen auszugleichen. Gewiss würde in solchen Fällen eine Begünstigung der Bedingungen des Herzfehlers nicht nur weitere Ernährungsstörungen nicht verhindern, sondern sogar das Herz zu einer grösseren Anstrengung reizen; diese Bedingungen dürfen aber nicht der ganzen Behandlung zu Grunde gelegt werden.

In dieser Mittheilung will ich nur über einige Beobachtungen berichten, die ich bei experimentellen und klinischen Untersuchungen mit einigen Arzneimitteln, welche die Herzfunction wieder herstellen sollen, gemacht habe.

Der Deutlichkeit wegen ist es unerlässlich, dass ich, wenn auch in aller Kürze, an einige physiologische Daten erinnere.

Wie bekannt, standen sich lange Jahre hindurch zwei Theorien der Herzfunction gegenüber. Die neurogene Theorie nimmt an, dass Reize und Coordination ausschliesslich durch intra- und extracardiale Nervenzellen und -Fasern zu Stande kommen, während die Mluskelfaser keine andere Eigensehaft haben soll, als die der Contractilität. Die myogene Theorie dagegen schreibt die ganze Function der Herzfaser zu, die ihre Thätigkeit auf Grund folgender fünf Eigenschaiten ausüben soll: weil sie erzeugt (Automatie), den Reiz empfängt (Erregbarkeit) und fortpflanzt (Leitvermögen), sich contrahirt (Contractilität) und einen gewissen Grad der Contraction beibehäl (Tonicität). Nervöse Einflüsse sollen nur insofern einwirken, als sie die Thätigkeit der Faser modifieiren und sie den verschiedenen Bedürfnissen des Organismus anpassen.

Diese letztere von Gaskell aufgestellte, von Engelmann²), Hering ${ }^{3}$ ) (der von Neuem Anhänger der myogenen Theorie geworden

1) Leclereq, Clinique du coeur. Paris 1908. 0. Doin.

2) Engelmann, Pflüger's Areh. 1894. Bd. LII. 1897. Bd. LXV.

3) H. E. Hering, Pflüger's Arch. 1901. Bd. LXXXVI. 1907. Bd. CXVI. Arch. f. d. ges, Phys. 1906. Bd. CXV. 
ist $^{1}$ ) u. $A$. verfochtene und ausgebildete Theorie haben bis vor einigen Jahren die meisten Autoren angenommen, und zuerst haben Wenkebach ${ }^{2}$ ) und Mackenzie ${ }^{3}$, dann auch Andere, ihre Daten bei der klinisehen Analyse der versehiedenen Arten von Pulsus irregularis verwendet. Dann wurde jedoch, wie bekannt, durch die Experimente Carlson's ${ }^{4}$ am Herzen von Limulus polyphemus und die durch neuere Untersuchungen Vilson's und der $\Lambda$ schoff'schen ${ }^{5}$ ) Schule bestaitigten und erweiterten anatomischen Untersuchungen Bethe's der Streit wieder angefacht, ob der Herzryhthmus im Muskel-oder Nervengewebe entstehe, und zu Gunsten der neurogenen Theorie entschieden. Die von den $\Lambda$ nhängern der myogenen Theorie angeführten Gründe ${ }^{6}$ ) wurden widerlegt und die neurogene Theorie wurde auf zwei wichtigen Grundlagen erbaut: dem Carlson'schen Experiment und dem Reichthum des IIerzens an Nervengewebe.

Aber auch von diesem Gesichtspunkte aus betrachtet ändern sich (ausser bei ihrem Entstehen) die speeifisehen Eigenschaften des Herzens nicht, nämlich seine Lrregbarkeit, sein Leitvermögen und der Kustand seines Tonus, die durch das intraeardiale Nervensystem bedingt sind, während die Contractilität die specifische ligensehaft der Herzmuskelfaser bleibt. Und da das von den extracardialen Nerven isolirte Herz fortfährt zu funetioniren, so muss man nothwendigerWeise annehmen, dass jene eher als Regulatoren wie als Urheber seiner 'Thätigkeit einwirken.

In pathologiseher Hinsicht muss die Veränderung der erwähnten extracardialen Nerven oine Verïnderung des Herzrhythmus zur Folge haben, die entschieden bedenklich wird, wonn ein schon verändertes Herz dadurch beeinflusst wird.

Doch abgeschen von der Erscheinung der Extrasystole, die durch eine Extrareizung bedingt ist, können Veränderungen der Eigenschaften des Herzens ausschliesslich nur von besonderen Verhältnissen der Faserzelle und des intracardialen Nervengewebes herrühren, da eine solche Störung sich nicht anders kundgeben kann, als durch mangelhaftes Fintreten der Funetion oder der Functionen, die beide zu erfüllen haben.

Daraus folgt für das Herz, dass hinsichtlich der Specifität und Intensität ein Parallelismus vorhanden ist zwischen der Läsion seiner Apparate und der Veränderung der betreffenden Ligenschaften, und dass

1) Cfr.: Abhandlungen d. Congresses zu Erlangen. 1910.

2) Wenkebach, Zeitschr. f. klin. Med. 1889. Bd. XXXVI. 1899. Bd. XXXVII. 1900. Bd. XXXIX und „Die Arhythmie als Ausdruck bestimmter Functionsstörungen des Herzens". Leipzig. 1903.

3) Mackenzie, The study of the pulse and of the movements of the heart. Edinburgh and London, Young J. Pentland.

4) Carlson, American journ. of phys. 1906. XII, I, XII.

5) Asch off, Deutsche med. Wochenschr. 1910, 2. (Referate.)

6) Cfr.: Von Cyon, Pflüger's Arch. 1906. Bd. CXIlI. 1907. Bd. CXVI. La Presse médicale, 15 mai 1907 und „Die Nerven des Herzens". Berlin. 1907. J. B. Hoffmann, Nagel's Handbuch der Phys. 1905. Bd. 1. - Mangold, Münch. med. Wochenschr. 1906. 
umgekehrt das Vorhandensein dieser Veränderungen uns anzeigt, in welcher seiner Functionen das Her'z nachgelassen hat.

Andererseits zeigt sich, da diese Eigenschaften des Herzens, auch schon deshalb, weil sie verschiedenen Apparaten angehören, von einander verschieden sind, je nachdem die eine mehr als die andere verändert ist, ein Complex von Erscheinungen, die von Fall zu Fall variiren und deshalb einem jeden ein ganz specielles Gepräge aufdrücken; es ist aber nicht möglich sie zu einem einzigen Krankheitsbilde zusammenzufassen oder auf ganz bestimmte Krankheitsursachen zurückzuführen, weil sie von Factoren abhängen, die von Fall zu Fall eintreten. Es giebt ja, wie bekannt, Fälle von Myocarditis, bei welchen die Herzaffection sich durch den Mangel an Erregbarkeit (batmotrope Störungen) oder an Leitvermögen (dromotrope Störungen) kundgiebt, während die Contractilität nicht nur als Eigenschaft, sondern auch als Kraft der Cuntraction vollkommen erhalten ist; dagegen giebt es Fälle, in welchen die Contractilität mangelhaft und die Kraft fast gleich Null ist (inotrope Störungen), während die Erregbarkeit vollständig erhalten und bisweilen sogar erhöht ist.

Dasselbe lässt sich hinsichtlich der mangelhaften Compensation sagen ${ }^{1}$ ): es giebt Fäle, in welchen (selbstverständich unter gleichen Vorbedingungen) seltene, langsame, aber verhältnissmässig starke Contractionen eintreten, ein deutliches Zeichen, dass die Erregbarkeit in höherem Grade beeinträchtigt ist und nach dem Bowditch'schen Gesetze die Reize, wenn sie sich summiren, so stark werden müssen, dass sie die Reizbarkeit wieder erregen, während die anderen Eigenschaften des Herzens erhaiten sind. Es giebt andere Fälle, in welchen, obgleich der Druck nicht niedrig ist, die Pulsationen häufig, klein und schwach auf einander folgen; hier ist natürlich die Contractilität und insbesondere dic Kraft der Contractionen beeinträchtigt. Endlich giebt es noch andere Fälle, und sie bilden die Mehrzahl, in welchen die gleichzeitige Veränderung aller oder fast aller Eigenschaften eintritt und häufig auch Extrareize sich einmischen, die das Bild durch Extrasystolen noch complicirter machen. Alsdann kann natürlich dic Veränderung einer jeden Eigenschaft nur durch eine specielle anatomisch-pathologische Veränderung der Herzapparate bedingt sein, und bei der Behandlung muss je nach den versehicdenen Fällen ein anderes Heilmittel angewendet werden. Von diesem Gesichtspunkte ausgehend soll man, so weit es möglich ist, nicht nur untersuchen, warum die Herzaffection eingetreten ist und wie sie sich kundgiebt, sondern auch ihre Intensität und ihre Bedeutung feststellen; dann kann man vernünftiger Weise das geeignete Mittel anwenden, ohne die Herzfaser oder das Nervensystem, deren Function sehon gesteigert ist, noch mehr zu reizen und zu riskiren, dass letztere erschöplt oder ganz aufgehoben wird.

Von den auf das Herz einwirkenden Mitteln habe ich bis jetzt Digitalis, Spartein, Convallamarin, Strychnin, Cacius grandiflora, Natriumbromid und -Jodid verwendet.

1) Cfr.: Mackenzie, I. c. und Les maladie du coeur. Paris 1911. A. Maloine. - Wenkebacb, 1. c. - Huchard, Maladies du coeur et des vaisseux. Paris 1899. - Riegel, Zeitschr.. f. klin. Med. Bd. XVIL. - Silbergleit, Ebenda. Bd. XXIX. - Grob, Deutsches Arch. f. klin. Med. Bd. XLII. 
Ich muss hier bemerken, dass die Angabe der batmotropen, dromotropen, tonotropen und inotropen Wirkung sich auf den Einfluss bezieht, den das Mittel auf Erregbarkeit, Tonicität und Contractilität des Herzens ausübt, und zwar nach Mlassgabe der im Obigen angeführten Grundsätze; der Zusatz positiv oder negativ bezeichner Zunahme bezw. Abnahme.

1. Digitalis. Wie bekannt, ist die Wirkung der Digitalis der Gegenstand zahlreicher Untersuchungen gewesen; dennoch kann man, wie Huchardl) mit Recht bemerkt, nicht von einer Uebereinstimmung bezüglich des Mechanismus ihrer Thätigkeit sprecken. Eine ganzo Reihe von Hypothesen and Theorien wurde von den versehiedenen Autoren aufgestellt, von Germain Sèéz) an, der jhre Wirkung durch ihre Anhäufung in den Herzganglien erklärte, bis zu Vulpian, der ohne Weiteres eine dirente Wirkung auf die Herzfaser annahm. Hirtz, Marey u. A. glaubten, sie wirke zunächst durch Zusammenziehung der peripherischen Gefässe ein und dann secundär auf das Centralorgan. In jüngster Zeit hat Leclereq ${ }^{3}$ ) diese Hypothese angenommen und sie seiner Herztherapie zu Grunde gelegt. Es ist in der That nicht einzusehen, dass ein Mittel gegen Herzleiden seine wohlthätige Wirkung ausübt, indem es die Hindernisse verstärkt, die in den meisten Fällen die Ursache der mangelhaften Compensation waren; das Secale cornutum ist ein gefüsszusammenziehendes Nittel, aber kein Heilmittel für das Herz. Bei Darreichung der Digitalis folgt Erhöhung des Druckes auf die Steigerung der Herzfunction oder fällt mit ihr zusammen, während die Hypotension in den Arterien messtens nicht dic Ursache der Läsion der Herzfaser ist, sondern auf sie folgt oder wenigstens mit ihr zusammentrifft. Marey hat nämlich constatirt, dass bei Abnahme des arteriellen Druckes eine damit parallel gehende Beschleunigung der Hersschläge eintritt, was beim Experiment eine wichtige biologische Forderung insofern erfüllt, als das Herz bestrebt ist, auf diese Weise die mangelhafte Ernährung der Gewebe auszugleichen. In klinischer Hinsicht stehen die Dinge aber anders, weil die beobachtete Hypotension in den Arterien (die Leclereq mit der Hypertension seiner Classificirung zu Grunde gelegt hat) schwerlich die. Wirkung von inotropen Störungen in der Faser ist oder nicht mit ihnen zuzammentrifft und deshalb der Ort ihres Entstehens im Myocardium zu suchen ist. Das Herzleiden würde nämlich zunehmen, wern man in derartigen Fällen den Druck erhöhte, ohne gleichzeitig auf die Contractilität des Herzens einzuwirken.

Wenn auch die nach Blutverlusten eintretende Tachycardic nur der Druckabnahme zuzuschreiben ist, so läst sich gewiss nicht dasselbe bei allgemeinen schweren Intoxicationen, namentlich bej solchen bakteriellen Ursprungs, annehmen.

Die Untersuchungen am suspendirten und noch mehr die am isolirten Herzen haben das Studium der Wirkung der Digitalis sehr gefördert und man kann im Allgemeinen sagen, dass ihre elective Wirkung auf die

1) Huchard, Le nuove consultazioni mediche, Hallardi. Milano.

2) Sèé, I. c. und. Académie de Mèd. de Paris, Janvier. 1882.

3) Leclercq, l. c. 
Herzfaser und das intracardiale Nervengewebe zur Evidenz nachgewiesen worden ist.

Braundenbo urg ${ }^{1}$ ) constatirte eine positive inotrope Wirkung, neben welcher eine flüchtige negative batmotrope Wirkung vorhanden ist, Braun und Mayer ${ }^{2}$ ) constatirten in einem ersten Zeitabschnit eine Zunahme der Frequenz des Rhythmus mit wachsender Energie der Systole, in einem zweiten Zeitabschnitt Abnahme der Zahl der Herzschläge.

O. Frank ${ }^{3}$ ) fand beim Studium der Dynamik des Ventrikels, dass in Fällen von Vergiftung durch Digitalis, wenn der Herzrhythmus schon auf die Hälfte seiner Frequenz herabgegangen ist, die Expansionscurve des isometrischen Maximums des normalen entspricht, während die Contractionen, wenn man sie für sich betrachtet, betrïichtlich vergrössert sind. Ausserdem sind die Expansionscurven des isotnoisehen Maximums vermindert. Nach Straub ${ }^{4}$ ) ist die Expansionscurve des isometrischen Maximums anscheinend normal, weil sie durch die $\Delta$ bnahme der Frequenz des Rhythmus ausgeglichen wird. Dicristina ${ }^{5}$ ) hat beobachtet, dass bei nicht stark toxischen Dosen positive inotrope und negative batmotrope Wirkung eintritt.

Vom klinischen Gesichtspunkt aus nimmt Hucha $\dot{r} d^{6}$ ) für die Digitalis eine doppelte Wirkung an, eine auf das Herz und eine diuretische. Er sagt, durch die erstere werde die Periode der Diastole verlängert und darauf trete Verstärkung der Systole ein; die diuretische Wirkung verursacht, wie er annimmt, zuerst eine Gefässzusammenziehung und dann eine Gefässerweiterung. Es ist jedoch logischer anzunehmen, dass dic diuretische Wirkung der Digitalis eine durch die Verbesserung der Herzfunction bedingte Wirkung der Wiederherstellung des normalen Gleichgewichts im Kreislaufe ist.

Die Zunahme der diastolischen Periode ist eine ausschliessliche Wirkung der Stärkung des Herzens und der, wenn auch in mässigem Grade stattfindenden, gleichzeitigen Abnahme der Erregbarkeit; wenn deshalb die Digitalis einorseits mit vollem Recht den ihr von Beau beigelegten Namen "Chinarinde des Herzens" verdient, so kann sie andererseits auch den ihr von Bouilland gegebenen "Opium des Herzens" beibehalten.

Dass ihre Wirkung sich vorzugsweise auf die Contractilität erstreckt, beweist auch der Unastand, dass bei dem (in Folge langsamer Phosphorvergiftung) in schwerer fetter Degeneration befindlichen Herzen, in welchem, wie bekannt, die Fibrillen in Bruchstücke zerfallen sind, ihre Wirkung fast gleich Null oder in anderer Weise schädlich ist in Folge des gleichzeitigen negativen batmotropen Einflusses, der in diesem Falle

1) Braundenbourg, Arch. f. Phys. 1904. Bd. IV.

2) Braun und Mayer, Sitzungsber. d. k. Akad. d. Wiss. Math.-naturw. Cl. CVIII, Abt. III.

3) O. Frank, Sitzungsber. d. Ges. f. Morphol. u. Physol. München 1897.

4) W. Straub, Zeitschr. f. exper, Pathol. u. Ther. 1905. - Arch. f. exper. Pathol. u. Pharm. Bd. XLV.

5) Dicristina, Journ. de Physol. et de Pathol. gén. 1908.

6) Huchard, loc. cit. 
auch auf veränderte intracardiale Nervenfasern und Ganglienzellen ausgeübt wird.

In einer Reihe von experimentellen Untersuchungen am isolirten Herzen fand ich diese Ansicht bestätigt und zwar sowohl in Bezug auf die jm Zeitabschnitt und in der von mir erwähnten Form eintretenden Functionen als auch hinsichtlich der Dynamik des Herzens. Unter dem Binfuss der Digitalis ist das Herz in Stande, eine grössere Arbeit zu leisten und eine grössere Anstrengung zu machen als unter normalen Verhältnissen; das Myocardium wird weniger erregbar und es sind immer stärkere Rejze nöthig, um eine Extrasystole hervorzurufen.

Dic Figuren 1 und 2 geben die von einem Herzen ron Emys europaea erhaltenen Cardiogramme wieder; das Herz wurde nach einer von mir vorgeschlagenen Hethode isolirt und suspendirt. Das erste Cardiogramm wurde vom Herzen aufgenommen, als letzteres noch keiner Behandlung unterzogen worden war, das zweite bei Einwirkung des Digitalins. Vergleicht man die beiden Höhen der systolischen Linie, so sieht man dentlich die Zunahme im zweiten Cardiogramm, in welchem die Höhe der extrasystolischen Linie zugenommen hat. Dje Reizschwelle hat jedoch abgenommen.

Bei einigen klinischen Untersuchungen hatte ich Gelegenheit, die Wirkung der Digitalis bei verschiedenen Herzaffectionen und Fällen von mangelhafter Compensation zu verfolgen, und konnte, wie mir schien, die Resultate der experimentellen Forschung klar bestätigt finden. Ich sah nämlich, dass sie nur in den Fällen ausgezeichnet wirkt, in welchen man das Contractionsvermögen und die Kraft der Contraction erhöhen und gleichzeitig die Erregbarkeit herabsetzen muss; die Wirkung ist viel ausgeprägter und wohlthätiger, wenn die Erregbarkeit erhoht ist. In allen anderen Fällen ist die Anwendung unnütz oder sehädlich.

$V$ on den zahlreichen von mir aufgenommenen Sphygmogrammen führe ich beispielsweise die von cinem Kranken an, der an Mitralstenose in der Periode mangelhafter Compensation litt.

Es handelte sich um einen 24 jährigen jungen Mann, bei dessen Anamnese sich nichts Bemerkenswerthos ergab ausser einer blenorrhagischen Infection, die er im Alter von 18 Jahren durchgemacht hatte. Er zeigte mässiges Oedem an den Beinen, mühsames Athmen und einen leichten Grad von Cyanose an den Lippen. Er hatte 120 Pulsschläge in der Minute. Aus Fig. 3 ersicht man, dass der Puls und die Grösse der einzelnen Pulswellen unregelmässig sind und letztere deshalb ron der Grösse der vorausgehenden Intervalle meistens unabhängig zu sein scheint, was nach Sahli') zu einer Herzinsufficienz in Bezichung gebracht werden muss. Der Puls ist im Allgemeinen ungleich, zcigt sich aber in gewissen Momenten entschieden als "Pulsus alternans".

Es ist also klar, dass im Herzen inotope und batmotrope Störungen vorhanden sind.

Zwei in Intervallen gemachte Injectionen von je $0,04 \mathrm{~g}$ Sparteinsulfat vergrössern die Welle des Arterienpulses, üben aber keinen Ein-

1) Sahii, Manuale dei metodi clivici. Vallardi, Milano 1911. 
fluss auf die Störungen der Erregbarkeit aus; es sind vielmehr Extrasystolen wahrzunehmen (Fig. 4).

Als ich dem Kranken Injectionen von Digitalone machte (4 von je $1 / 2 \mathrm{ccm}$ in 2 Tagen), erhielt ich die in Fig. 5 dargestellte Curve, wobei sich das Allgemeinbefinden des Patienten, dem übrigens alle Pflege zu Theil wurde, die der Fall erforderte, rasch besserte.

Aus allen diesen Beobachtungen würde sich also ergeben, dass der gleich wohlthätige Einfluss der Digitalis auf die Contractilität und auf die Erregbarkeit des Herzens die Stärkung des Jetzteren (Zunahme der Contractilität und Kraft der Contraction) zur Folge hat, Zunahme der systolischen und diastolischen Periode (in Folge der grösseren Langsamkeit, mit der die Contraction erfolgt, da Erregbarkeit und Leitvermögen vermindert sind), und daraus sich ergebende Verminderung der Herzschläge; dem zu Folge wird mit der Zunahme des Druckes und durch die Regulirung der Hydraulik des Kreislaufes die Harnsecretion wieder in dic Wege geleitet.

Spartein. Es fehlt an wichtigen Untersuchungen aus neuerer Zeit über den Einfluss des Sparteins auf das Herz und namentlich über den Mechanismus seiner Wirkung; in den bis jetzt vorliegenden stimmen die Ansichten nicht überein. Seé rechnet es unter die Tonica des Herzens und Dieulafoy ${ }^{1}$ ) stimmt ihm bei.

Dicristina ${ }^{2}$, der am suspendirten Froschherzen experimentirte, fand, dass das Sparteinsulfat, wie das Digitalin, wenn es in ziemlich starker Dosis dargereicht wird, die Arbeit des Herzens beeinflusst, indem es seine systolische Phase verlängert, die sofort energischer wird. Die verschiedenen Phasen des Blutumlaufs im Herzen werden länger, nicht weil Störungen in der Uebermittlung der spontanen Reizung eintreten, sondern weil die Contraction der Faserzelle langsamer erfolgt; unter der Einwirkung des Sparteins wird das normale Herz weniger erregbar. Auf das degenerirte Herz übt das Spartein, wie das Digitalin, keinen Einfluss aus.

Leclereq hält das Spartein für ein neuromusculäres Tonicum des Herzens, dessen Wirkung fast identisch mit der des Digitalins ist; beide sollen sich unterscheiden durch die Geschwindigkeit ihrer Wirkung und insbesondere durch die Regelmässigkeit und grössere Beschleunigung der Herzschläge, die das Spartein bewirkt.

Aus meinen experimentellen Untersuchungen ergiebt sich dagegen, dass das Spartein allerdings eine schnellere, aber weniger andauernde Wirkung auf die Contractilität ausübt als die Digitalis; aber die Erregbarkeit nicht beeinflusst. Wird ein isolirtes Herz von Lmys europaea mit Spartein behandelt, so nimmt die Zahl der Herzschläge etwas ab, weil die Systole sich ausdehnt und die Diastole weiter wird. Die Reizschwelle wird nur dann modificirt, wenn das Herz nachzulassen beginnt, wie in den Fällen, in denen es gar keiner Behandlung unterzogen wird.

Vom klinischen Gesichtspunkte aus habe ich ebenfalls Beweise davon erhalten; jch erinnere an das bei einem mit Digitalis behandelten Kranken

1) Dieulafoy, Patologia interna. Vallardi, Milano.

2) Dicristina, loc. cit. 
während einer Periode mangelhafter Compensation aufgenommene Sphygmogramm, bei welchem die Darreichung des Sparteins die Welle des Arterienpulses erhöht, aber den Erregbarkeitszustand nicht beeinflusst hatte. Fig. 6 stellt ein Sphygmogramm dar, das bei einer Kranken aufgenommen wurde, die an Mitralinsufficienz litt, und zwar in der Periode der mangelhaften Compensation. Hier bandelte es sich um eine 28 jährige Frau, die schon mehrere Anfälle von acutem Gelenkrheumatismus erlitten hatte. Der Puls war selten, träg und klein. Es waren offenbar negative inotrope und batmotrope Herzstörungen vorhanden. Die Welle des Arterienpulses war klein, der Druck niedrig und in Lebcreinstimmung mit den in letater Zeit von Sahli gemachten Beobachtungen betrug die Zahl der Pulsschläge 50 in der Minute (Fig. 6).

Drei Injectionen von Coffein hoben die Frequenz des Pulses, aber die Höhe der Welle ist noch geringer (Fig. 7), was offenbar beweist, dass das Mittel auf die Erregbarkeit und nicht auf die Kraft der Contraction des Herzens eingewirkt hat.

In der Folge wurden zunächst abwechselnd lnjectionen von Spartein und Coffein, dann von Spartein allein gemacht, worauf der Puls die in Fig. 8 dargestellte Form erreichte, bei der, wie man sieht, der Puls häufiger und die Contraction kräftiger ist.

Convallamarin und Convallarin. Das gebräuchlichste von ihnen ist das Convallamarin; sie haben gleiche Wirkung, doch ist die des ersteren nicht nur eine schnellere, sondern auch eine entschieden energischere. Maraglianol) fand, dass das Convallamarin in gewissen Fällen eine stärkere Wirkung ausübte als das Digitalin. Bogojanski²) bemerkt in seiner in der Klinik Botkin's angefertigten experimentellen und klinischen Arbeit, er habe beobachtet, dass die Wirkung der Convallaria majalis vollständig bei Fröschen eintreten kann, auch wenn die Vagi vorher durchschnitten oder durch Atropin gelährnt wurden, oder wenn das Centralnervensystem zerstört worden ist; nur musste im letzteren Falle die Dosis des Mittels stärker sein. $Z u$ den gleichen Resultaten gelangten Troitski ${ }^{3}$ ) und Isaier ${ }^{4}$ ), als sie die Wirkung der Convallamarina studirten, sie beobachteten bei Fröschen, dass, auch wenn das Herz in Folge der Einwirkung dieses Mittels zum Stillstand gebracht worden war, die Erregbarkeit und die Reflexbewegungen noch eine gewisse Zeit hindurch erhalten blieben, und dass Durchschncidung der Vagi, ihre Juähmung durch Atropin, Durchschneidung des Hirn-Rückenmarks und Isolirung des Herzens von den Nervencentren, die durch das Convallamarin hervorgerufenen Erscheinungen nicht modificirten. Rummo und Andrea Ferranini ${ }^{5}$ ) classificiren es als ein Myo-Cardio-Cineticum. Maurel6) schliesst aus seinen Experimenten, dass es ein gefässzusammenziehendes und den Kreislauf beschleunigendes Mittel ist.

1) Maragliano, Boll. della Clinica Medica di Genova.

2) Bogojanski, Wratsch. 1880. No. 49.

3) Troitzki, Wratsch. 1889. No. 47. 1881. No. 15. 1882. No. 18, 40, 41.

4) Isaiev, Medizinskaia Gazetta. 1881. No. 10.

5) Rummo e Ferrannini A., Riforma Medica. 1888.

6) Maurel, Comptes rendus de l'acad. de Méd. Séances, 7.-21. juillet 1906. 
Aus einer Reihe von Experimenten, die ich vor einigen Jahren gemacht habe'), konnte ich die Schlussfolgerung ziehen, dass die Glucoside von Convallaria majalis die Triebkraft des Herzens steigern, indem sie die Systole verstärken und die Erregbarkeit des Hyocardiums erhöhen. Diese letztere ligenschaft gewinnt eine hohe Bedeutung, wenn das Her' der fettigen Entartung anheimgefallen ist.

Denn beim fettig entarteten Herzen, auf das die Digitalis gar nicht oder höchstens schädlich einwirkt, erfolgt bei Behandlung mit den Glucosiden der Convallaria majalis Zunahme der Zahl der Herzschläge, Hebung der Systole und Verlängerung der Diastole; das Leben des Herzens dauert länger und der Rhythmus, der sich bei den keiner Behandlung unterzogenen degenerirten Herzen leicht ändert, erhält sich gleichmässig. Die Reizschwelle nimmt zu.

Ich habe das Convallamarin in einem Falle von mangelhafter Compensation angewendet, in welchem nicht nur die Contractilität, sondern auch die Erregbarkeit offenbar vermindert war, und zwar mit ausgezeichnetem Erfolg.

Wenn wir die aus den Resultaten der Experimente und aus der klinischen Beobachtung gezogenen Schlüsse zusammenfassen, so können wir sagen, dass das Convallamarin eine positive inotrope und eine positive bathmotrope Wirkung ausübt.

Strychnin. Die bei klinischen und experimentellen Untersuchungen erhaltenen Resultate stimmen nicht übercin. weshalb die bezüglich des Mechanismus der Wirkung des Strychnins aufgestellten Hypothesen von verschiedener Art sind.

Bayldon²) fand bei mit Strychnin vergifteten Katzen nach dem Tode, dass das Herz bei einigen unbeweglich und nicht mehr erregbar war, bei anderen dagegen noch pulsirte.

Ambrosoli ${ }^{3}$ ) nahm an, dass bei Anwendung des Alkaloids zuerst Zunahme und dann Abnahme der Zahl der Herzschläge eintritt; 'T'aylor glaubte, es übe auf das Herz eine ähnliche Wirkung aus wie auf die übrige Musculatur des Körpers; Richter ${ }^{4}$ ) dagegen lengnete jede direkte Wirkung auf das Herz, dessen Zustand durch die spasmodische Contraction der kleinen Gefässe bedingt wäre. Diese Ansicht wurde von den späteren Autoren $\left[\right.$ Heinemann ${ }^{5}$ ), Stciner ${ }^{6}$ ), Foleh ${ }^{7}$ ), Möller ${ }^{8}$ ), Lövit ${ }^{9}$ ) ete.] nicht getheilt, die dom Herzen die Verlangsamung des Pulses zuschreiben, die man bei den der Wirkung des Strychnins ausgesetzten Warmblütern beobachtet. Vol. VIII.

1) La Franca, Archivio di Farmacologia sperimentale e Scienze affini. 1907.

2) Bayldon, Lancet. 1856.

3) $\Lambda$ mbrosoli, Gazzetta Lombarda. 1856.

4) Richter, Zeitschr. f. ration. Med. 1863. Bd. XVIII.

5) Heinemann, Virchow's Arch. 1865. Bd. 33.

6) Steiner, Arch. f. Anat. u. Phys. Abtheil. 1874.

7) Folch, Vierteljahrssehr. f. gerichtl. Med. 1874. No. 7. Bd. XX.

8) Möller, Ugeskr. f. Jäg. 3 R. Bd. XVII.

9) Lövit, Pflüger's Arch. f. d. ges. Phys. 1882. Bd. XXVIII. 
Brunton ${ }^{1}$, der die Frage gelegentlich behandelt, sagt, die Pulsationen seien während der Anfälle schlaffer, aber im Allgemeinen wirke das Strychnin als Reizmittel auf das llerz ein; or orinnert an die von ihm gemeinschaftlich mit $\mathrm{Cash}^{2}$ ) ausgeführten Lntersuchungen; bei denen sie den Stannius'schen Stillstand nach Injection von Strychnin ins Innere des Ventrikels beobachten konnten, während sie in dem mit Strychnin behandelten Herzen nach der Stannius'schen ligatur keinen Stillstand eintreten sahen.

Hebdom ${ }^{3}$ ) sah, als er am isolirten Säugethierherzen experimentirte, dass kleine Dosen von Strychnin Abnahme der Zahl und Weite der Herzschläge bewirken, mittiere sie erhöhen, starke sie herabsetzen und dann Stillstand verursachen; auf diesen Stillstand beruft sich Verworn ${ }^{4}$, wenn er glaubt, auf Lähmung des Herzens folge die vollständige Iuähmung des Markes. Igersheimer ${ }^{5}$ ) hat in jüngster Zcit aus seinen Experimenten gefolgert, dass im Mllgemeinen bei Strychninvergiftung der motorische Muskelapparat der Ort der 'Thätigkeit im Herzen ist, und dass die Stillstände in keinem Causalnexus zu den centralen Lähmungen stehen.

In einer früheren Arbeit $\left.{ }^{6}\right)$ habe ich über dic Resultate berichtet, die ich bei einigen Experimenten über die Wirkung des Strychnins erhalten habe, und die darin bestanden, dass das Herz unter der Einwirkung des Alkaloids sich in einem ersten Zeitabschnitt in einem Zustand gesteigerter Erregbarkeit befindet, der in einem zweiten wieder herabgeht; in dieser Periode wird das Herz (wenn die Dosis hoch ist) leicht arhythmisch, wahrscheinlich infolge von Störungen der Erregbarkeit, die in der Herzfaser eingetreten sind. Dieses Verhalten zeigt auch noch das degenerirte Herz, in welchem während eines ersten Zeitabschnittes Zunahme der Herzschläge, dann Abnahme derselben zu bemerken ist. Dic Reizscbwelle ist in einem ersten Zeitabsehnitt stets erhöht und das degenerirte Her\% reagirt immer auf die linwirkung elektrischer Reize durch eine Reihe von Wellenlinien.

Wie bekannt, wird das Strychnin in der Klinik, und zwar mit Nutzen, jedes Mal verwendet, wenn das Her', obwohl es an Kraft nicht eingebüsst hat, sich infolge mangelhafter Krregbarkeit oder J Jeitfähigkeit zu langsam entleert. Von diesem Gesichtspunkt aus ist anzunehmen, dass das Strychnin eine positive bathmotrope und dromotrope Wirkung ausübt.

Bei Anwendung des Strychnins ist es ton Wichtigkeit die Dosis genau festzustellen, da kleine Dosen, wie es scheint, ungrenügend sind, um ihren Zweck, die Wicderbelebung der Function, zu erreichen, wie aueh grosse Dosen Jähmend wirken in Gegensatz zu den mittleren, die erregend einwirken.

Cactus grandiflora. Wie bekannt, wird von dieser Pflanze das

1) Brunton, A. textbook of pharmacology etc. 1885.

2) Cash, St. Barth. Hlosp. Rep. 1880.

3) Hebdom, Skandinar. Arch. f. Phys. 1899.

4) Verworn, Arch. f. exp. Path. und Pharm. 1900. Bd. 54.

5) Igersheimer, Arch. f. exp. Path. u. Pharm. 1906. Bd. 54.

6) La Franca, l. c. 
flüssige Extract und die Muttertinetur verwendet; auch hat man das Cactin zu Heilzwecken gebraucht. Orland Jones ${ }^{1}$ ), der im Jahre 1888 die Aufmerksamkeit auf dieses Mittel hinlenkte, fand, obwohl er mit Recht bemerkte, es sei nicht bei allen Herzleiden indicirt, dass es ein gutes Cardiocineticum ist. Auld ${ }^{2}$ ) räth, es als Ersatz für das Digitalin zu verwenden oder um dessen cumulative Wirkung zu vermeiden, wie auch wenn das Digitalin versagt. Das gleiche Urtheil fällen Myers, Wilcox ${ }^{3}$ ) und Ellingwood ${ }^{4}$ ). Boy Tessier und Boinet5) fanden, dass grosse Dosen die Herzfunction verlangsamen, hleine sie beschleunigen. Williams und Hills sind der Ansicht, dass der Cactus von Nutzen ist bei Functionsstörungen des Herzens und bei reflexivem Herzklopfen infolge ehronischer Tabakvergiftung.

Gibellib) vergleicht die vor und nach der Behandlung bei verschiedenen Kranken (Aorteninsufficienz, Basedow'sche Krankheit, Rheumatismus, Chorea minor, Anämie) entnommenen Sphygmogramme und kommt zu dem Sehluss, dass das Mittel Zunahme der Höhe und Kraft des Pulses verursacht, die er nicht in Beziehung zur Erhöhung des Blutdruckes bringt, sondern zur verminderten Spannung in den Gefässen, die unter dem Einfluss der Blutwelle stärker erweitert werden und einen ausgeprägten Dicrotismus zeigen. Der Cactus übt eine Wirkung auf die Arhythmie des Pulses aus, indem er letzteren in der Mehrzahl der Fälle rhythmisch macht, aber seine Frequenz nicht verändert.

Dambrosio ${ }^{7}$ ) berichtet in einer vor Kurzem veröffentlichten Arbeit, er habe gefunden, dass subcutan und intravenös injicirtes Cactin bei Fröschen und Kaninchen keine Zusammenziehung der peripherischen Gefässe bewirkt, bei Hunden intravenös injicirt Erböhung des Blutdrucks zur Folge hat. Bei Herzleidenden verstärkt es die systolische Energie des Myocardiums, erböht die Frequenz der Herzfunetion, verkürzt die Systole und die Diastole, macht den Puls häufiger und stark, erhöht fast immer den arteriellen Druck und wirkt leicht harntreibend. Er hält es für indicirt in allen Fällen, in welchen die Systole verstärkt werden muss, also vorzugsweise bei Aortafehlern, Myocarditis und Herzschwäche, für contraindicirt dagegen bei Mitralfehlern und Fehlern der mangelhaften Compensation.

Bei Experimenten am isolirten normalen Herzen fand ich Verlängerung der Systole und Zunahme der Diastole an Weite. Die Reizschwelle sinkt zuelst und erhält sich dann unverändert. Im degenerirten Herzen war die Wirkang des Mittels ganz unbedeutend.

In zwei Fällen von Myocarditis, in denen die Störungen ausschliesslich die Contractilität betrafen, während die Erregbarkeit etwas erhöht

1) Jones, Semaine méd. 1888.

2) Aulde, Ther. Gaz. 1891.

3) Wilcox, Post. Graduate, Clin. Society. 1891.

4) Ellingwood, New York. Med. Rec. 1905.

5) Boinet et Boy Tessier, Bull. gón. de Thér. 1891.

6) Gibelli, Gazz. degli Osped. Anno XV.

7) Dambrosio, Gazz. intern, di med. Chir. ed Igiene. 1910. 
war, zeigten sich stärkere Contractionen und die Höhe der systolischen Linie war gestiegen, während die Frequenz sehr wenig beeinflusst war. In einem Falle von mangelhafter Compensation in Folge von Aorteninsufficienz, bei dem Störungen der Contractilität und der Erregbarkeit vorhanden waren, stärkte das Cactin die Herzthätigkeit und erhöhte die systolische Welle; der Puls aber wurde träge und noch seltener und es musste gleichzeitig Strychnin und Coffein angewendet werden, um die Erregbarkeit des Herzens wiederherzustellen. Fig. 9 zeigt ein Sphygmogramm, das bei einem Kranken aufgenommen wurde, der an chronischer Myocarditis litt. Der Fall betraf einen 34jährigen Mann, der an Syphilis gelitten und ein sehr mühsames Leben geführt hatte. Wie man sieht, waren beträchtliche Störungen der Erregbarkeit vorhanden. Der Kranke wurde mit Cactus grandiflora (flüssigem Extract) behandelt; nach fünf Tagen wurde der Puls regelmässiger und die Contractilität zeigte ein weniger ungeordnetes Verhalten, wobei jedoch Erscheinungen von Extrasystole andauerten, die in der in Fig, 10 dargestellten Curve deutlich sichtbar sind.

Dann wurde dem Kranken ausser dem Cactin gleichzeitig auch Natriumjodid und Bromid dargereicht, worauf der Puls nach weiteren 6 Tagen das in Fig. 11 dargestellte Sphygmogramm ergab.

Wie man leicht aus den von mir angeführten Beobachtungen ersieht, lässt sich mit Sicherheit annehmen, dass der Cactus auf die Contractilität des Herzens einwirkt und dass die Anwendung dieses Mittels Zunahme der systolischen Kraft und demgemäss eine grössere Weite der Arterienwelle zur Folge hat. Bei allen Patienten, bei denen ich es anwandte, erhielt ich genau die gleichen Resultate. Was seinen Einfluss auf die Erregbarkeit anbelangt, so kann man eine negative bathmotrope Wirkung annehmen, die aber sehr leicht, kaum wahrnehmbar ist.

Man kann also sagen, dass die Wirkung des Cactus eine positive inotrope und eine leicht negative bathmotrope ist; was die Erhöhung des Blutdruckes betrifft, so spricht alles für die Annahme, dass sie durch die gesteigerte Energie des Herzens oder durch eine gleichzeitige Reizung des vasomotorischen Centrums verursacht wird.

Coffein. Es ist gewiss nicht nöthig, hier auch nur einen Ueberblick über die versehiedenen Hypothesen zu geben, die aufgestellt worden sind, um den Mechanismus der Wirkung des Coffeins zu erklären; sie sind zahlreich und hinlänglich bekannt. Wie bekannt, ist das Coffein ein Reizmittel für das Nervensystem überbaupt und das des Herzens und wirkt auch als Diureticum. Rummo ${ }^{1}$ ) glaubt, es wirke indirect auf das Myocardium ein, indem es die Herzganglien und das Herzcentrum des Bulbus erregt. Sèé ${ }^{2}$ ) rechnet es zu den nur diuretischen Arzneimitteln, und Dieulafoye ist derselben Ansicht. Cervello erklärt die diuretische Wirkung durch eine Erweiterung der Gefässe, die sich vielleicht auch auf die Nieren ausdehne.

Beim isolirten Herzen wirkt es hauptsächlich aul die Erregbarkeit

1) Rummo, Formulario terapeutico. Napoli.

2) Sèé, loc. cit. 
des Myocardiums ein, die unter seinem Einfluss zunimmt, um dann wieder abzunehmen. Die Erequenz der Pulsationen nimmt zu und nur bei den ersten steigt die systolische Linie zu grüsserer Höhe an. Auch im degenerirten Herzen liegt die Reizschwelle höher und bei Anwendung mässiger Dosen wird das Leben des Herzens länger erhalten.

Bei Besprechung des Sparteins habe ich ein Sphygmogramm gebracht, das ich während der Periode der mangelhaften Compensation bei einem an Mitralinsufficienz leidenden Kranken aufgenommen hatte; das Coffein hatte in diesem Falle einen positiven Einfluss auf die Erregbarkeit des Herzens ausgeübt, aber gleichzeitig die Contractilitätsbedingungen nicht beeinflusst (Fig. 6 und 7).

In allen Fällen, in welchen die mangelhafte Herzthätigkeit sich durch ungenügende Kraft der Contraction kund giebt, während der Rhythmus häufig ist, wird dieser Zustand des Herzens durch das Coffein nicht nur nicht gebessert, sondern die Verhältnisse werden bald schlimmer, wenn das Mittel auch eine gewisse Zeit lang wohlthätig einzuwirken scheint, und es tritt leicht Erschöpfung des Herzens ein.

Das Coffein hat also, wie auch die meisten Autoren annehmen, eine positive bathmotrope und höchstwahrscheinlich auch eine dromotrope Wirkung.

Natriumbromid. Die Verwendung der Bromide bei Herzaffectionen, namentlich bei solchen nervösen Ursprungs, ist ganz allgemein; ihre beruhigende Wirkung erstreckt sich nämlich auch auf das Herz. Die neueren Untersuchungen über die Beziehung zwischen den Herzschlägen und den im Blut enthaltenen anorganiscben Salzen haben sehr wichtige Thatsachen zur Evidenz nachgewiesen, aus denen die Klinik, wie mir scheint, Nutzen ziehen kann. Zu erwähnen sind hier die Arbeiten von Gaule, Volkmann, Magnus, Martius, Ringer, Locke, Green, Howell, Göthlin und Langendorffl) über dieses Thema; sie haben im allgemeinen festgestellt, welche Stoffe in der Nährflüssigkeit des Herzens vorhanden sein müssen. Unter den verschiedenen Autoren waren es jedoch $\mathrm{Howell}^{2}$ ) und $\mathrm{Locb}^{3}$ ), die das Problem von einem neuen und auch bestimmteren Gesichtspunkte aus behandelt haben. Ersterer ist der Ansicht, dass die stimulirende Wirkung durch das Ca-Ion bedingt ist, während letzterer dagegen dem Na-Ion die grösste Bedontung zuschreibt.

Lingle ${ }^{4}$, der an Muskelstreifen des Herzens von Emys europaea experimentirte, hat die Ansicht Loeb's bestitigt; Martius's aber und später Benedict6) zogen die Schlussfolgerung (wobei sie sich auch auf die Ansichten Howell's beriefen), dass Natrium und Calcium nöthig

1) Cfr.: Magnus, Arch. f. exper. Pathol. u. Pharm. 1901. - Green, Amer. Journ. of Phys. 1898. - Göthlin, Skandinav. Archiv f. Phys. 1902. - Langen dorff, Ergebn. d. Physiol. 1902. Jahrg. I. Abth. II.

2) Howell, Amer. Journ. of Physiol. 1898-1901.

3) Lo eb, Festschr. f. Prof. Dr. A. Frek, 1898.

4) Lingle, Amer. Journ. of Phys. 1900-1902.

5) Martius, Ibid. 1904.

6) Benedict, Ibid. 1905. 
sind, wenn der Herzrhythmus entwickelt und erhalten werden soll. Diese Theorie, welche die beiden früheren vereint, ist von Benedict folgendermaassen formulirt worden: Die Erzeugung des Heryrhythmus ist durch die Wirkung des Anions des verwendeten Salzes bedingt, während die Hauptaufgabe des Kations dem Anschein nach darin besteht, den Tonus des Herzmuskels zu erhalten.

Auch wenn man keinen so deutlichen Unterschied zwischen der Wirkung des Anions und der des Kations annehmen will, kann man doch der Ansicht sein, dass beide Arten von Ionen einen verschiedenen Einfluss auf die fundamentalen Eigenschaften der Herzelemente ausüben.

Bei Experimenten am isolirten Herzen von Emys europaea habe ich gefunden ${ }^{1}$ ), dass die Br-Jonen, wenn sie in der das Herz bespülenden Flüssigkeit in richtigem Verhältniss vorhanden sind, genügen um die Erregbarkeit dieses Organs zu erhalten, das in einer $\mathrm{NaBr}$ enthaltenden Flüssigkeit viel länger weiter pulsirt als ein von reiner Ringer'scher Flüssigkeit bespültes Herz. Die Reizschwelle erhält sich nämlich auf einer beträchtlichen Höhe; die Daner der refractären Periode ändert sich aber nicht, während die Latenzzeit der Reizung mit der Zeit etwas länger wird.

Weitere klinische Untersuchungen können dazu dienen, diese Resultate auf dem Gebjete der menseblichen Pathologie zu controliren, um so mehr als der wohithätige Einfluss, den das Bromid bei verschiedenen Herzaffectionen ausübt, ein positives Ergebniss dieser Untersuchungen erwarten lässt.

Beim gegenwärtigen Stand unserer Kenntnisse könnte man denken, dass das Bromid auf die Erregbarkeit einwirkt, indem es sie herabsetzt, wenn sie übermässig gross ist, aber sie erhält, wenn sie normal ist. Im ersten Falle würde es auf die extracardialen Nerven wie anf das übrige Nervensystem, im zweiten direct auf den intracardialen Nervenapparat einwirken.

Bei Besprechung der Wirkung des Cactus habe ich erwähnt, dass ich bei einem Kranken die Beobachtung machte, dass das Bromid und das Jodid die Erregbarkeit regulirten; sie setzten aber ihren Index nicht herab.

Da jhre Wirkung durch das Br-Ion bedingt ist, so muss man natürlich annehmen, dass alle oder fast alle Bromide ihren Einfluss auf dieselbe Weise ausüben, wenn nieht etwa die betreffende Verbindung an und für sich toxisch ist.

Die gleiche, aber nicht so entschiedene Wirkung habe ich beim $\mathrm{Na} I$ gefunden.

\section{Zusammenfassung und Schlussfolgerungen.}

Wenn ich das Gesagte kurz zusammenfasse, kann ich sagen, dass ein jedes der erwähnten Arzneimittel, wie aus den mitgetheilten Beobachtungen hervorgeht, auf den intracardialen Nervenapparat oder auf

1) La Franca, Archivio de Fisiologia, 1a, 2a, 3a. Comunicazione. 1909. Vol. VI, Fasc. V.-VI. 
die Faserzelle bezw. auf beide gleichzeitig einwirkt, indem es die betreffenden Functionen modificirt; die Untersuchung der letzteren ermöglicht es, die speciellen Verhältnisse der Herzaffection festzustellen. Die Digitalis übt eine positive inotrope und eine negative bathmotrope, vielleicht auch eine negative dromotrope Wirkung aus; das Spartein hat sicher eine positive inotrope Wirkung; das Convallamarin zeigt eine positive inotrope und eine positive batmotrope Wirkung; der Cactus hat positive inotrope und leichte negative bathmotrope Wirkung; das Strychnin hat negative bathmotrope und negative dromotrope Wirkung; das Coffein positive bathmotrope und auch vielleicht positive dromotrope Wirkung; das Bromid und auch, aber in leichterem Grade, das Natriumjodid, haben bathmotrope und dromotrope Wirkung in dem Sinne, dass sie die Erregbarkeit und die Leitfähigkeit erhalten. .

Ich stelle diese Schlussfolgerungen in nachstehender Tabelle zusammen:

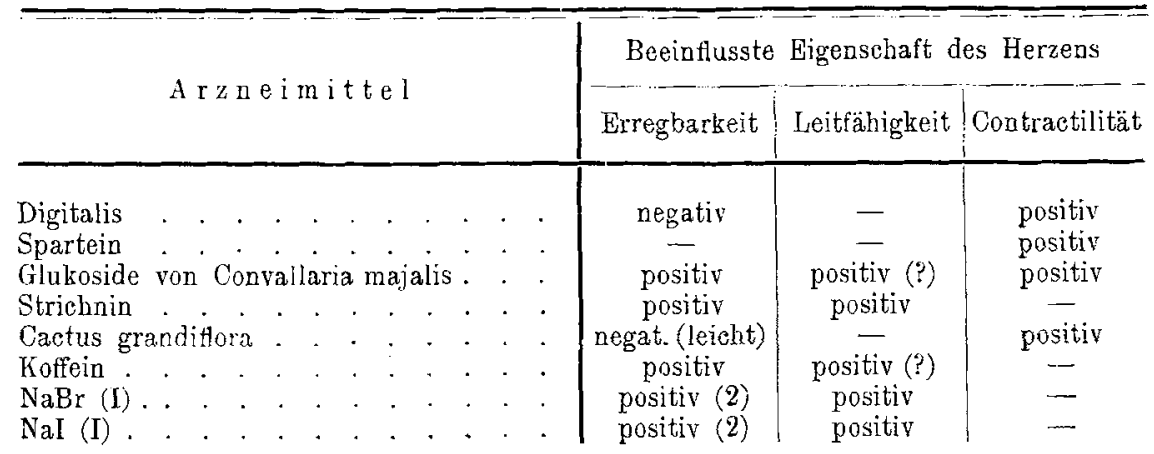

(I) Betrifft die Darreichung von mittleren Dosen.

(2) In dem früher festgestellten Sinne, nämlich dem der Erhaltung der Erregbarkeit.

lch glaube nicht, dass meine Beobachtungen, namentlich die wenigen klinischer Art, hinreichen, um jetzt schon eine entschiedene Anwendung dieser Grundsätze za rechtfertigen, da noch weitere Studien erforderlich sind um ihren Werth endgültig zu erweisen; es scheint mir jedoch, dass sie die wahrhaft rationelle Art und Weise angeben, wie man mit Erfolg den Kranken, nicht die Herzaffection, heilen kann.

Gewiss entsteht hier eine ziemlich schwierige Frage, namentlich in der Privatpraxis, in der man nicht immer über passende Apparate verfügt, nämlich die genaue Abschätzung der Veränderungen der Herzfunction; ein Aussetzen des Pulses kann durch eine Veränderung der Contractilität, der Erregbarkeit oder der Leitfähigkeit bedingt sein, kann aber auch die Wirkung extracardialer Einflüsse sein. Man kann jedoch sagen, dass gewöhnlich die allgemeinen Symptome und die der Herzfunction, wenn man sie zu denen des Pulses in Beziehung bringt, Kriterien ergeben können, die zur Erklärung der Verhältnisse der Herzfaserzelle ansreichen. 